Original Research Paper

\title{
Analysis and Mechanical Design Solutions for Sit-To-Stand Assisting Devices
}

\author{
Pierluigi Rea and Erika Ottaviano \\ DICeM: Department of Civil and Mechanical Engineering, \\ University of Cassino and Southern Lazio, via G. Di Biasio 43, 03043, Cassino (FR), Italy
}

\author{
Article history \\ Received: 28-11-2016 \\ Revised: 03-12-2016 \\ Accepted: 10-12-2016 \\ Corresponding Author: \\ Erika Ottaviano \\ DICeM: Department of Civil \\ and Mechanical Engineering, \\ University of Cassino and \\ Southern Lazio, via G. Di \\ Biasio 43, 03043, Cassino \\ (FR), Italy \\ Email: ottaviano@unicas.it
}

\begin{abstract}
Sit-to-stand can be considered the most common daily-life activity and it can be defined as a change of posture, in which the base of support is transferred from the seat to the feet. It requires both voluntary movement of different body segments that contribute to the equilibrium and control during an important displacement of the Centre Of Gravity of the body. This activity can be considered of crucial importance for elderly and people with reduced mobility to achieve minimal independence in daily-life at home. In this study, we present and compare three design solutions for the support mechanisms to be used in assisting mechatronic devices. The reported solutions and considerations are supported by experimental activity, which was carried out during trials to track and record trajectories and the orientation of the trunk of the body during the sit-to-stand.
\end{abstract}

Keywords: Assistive and Rehabilitation Engineering, Mechanical Design, Assisting Device, Simulation, Experimental Evaluation

\section{Introduction}

A reduced mobility due to physiological degradation of the motor system by aging or neurological diseases can have widespread, detrimental effects for older adults and ultimately accelerate the process of ageing. Adults for whom mobility is a problem certainly experience a reduction in the quality of their social life. Recent studies reports that the proportion of Europeans aged 65 years and older will grow from $16 \%$ in 2000 to $24 \%$ by 2030 , as reported by EIU (2011). Life expectancy is also increasing, particularly in the richer European countries. Despite the good news of higher life expectancy, older people are more likely to be prey to chronic disease. In 2010, over one-third of Europe's population is estimated to have developed at least one chronic disease. Several factors have an adverse effect on mobility, the most obvious being physical impairments, loss or reduction of visual and auditory ability and of the key function of balance. Those factors greatly influence the capability of those persons to perform daily-life activity in autonomy. It is obvious that being autonomous and living in the well-known and familiar home for as long as possible is an invaluable increase in living quality as one gets older. Among several daily-life activities the most common is the sit-to-stand that permits the changing of posture from the seated to the standing ones and vice-versa.
Some devices have been conceived to assist people in daily-life activities such as walking (Palopoli et al., 2015), picking objects (Graf et al., 2004), aiding during the sit-to-stand (Nagai et al., 2003; Hirata et al., 2008), or for human-robot interaction (Schiffer et al., 2010). Overviews reporting existing aiding mobility devices are given in (Martins et al., 2012; Krishnan and Pugazhenthi, 2014). The concept of using exoskeletons to assist caregivers was proposed in (Yoshimitsu and Yamamoto, 2004). Some interesting mechatronic systems are already commercially available, such as TTW (2016) and SSB (2016) in which the concept of "easy to adjust to height of the subject" is fulfilled. A mechatronic device for the inversion therapy was developed by (Rea et al., 2013b). In this paper, we compare solutions for the design of support mechanisms to be used in assisting devices, starting from the same sit-to-stand motion, which was executed during an experimental trial.

\section{Experimental Evaluation of the STS}

Motion analysis deals with human motion detection and motion tracking. Recently, there is a fast development of reliable, easy-to-use and economic systems to monitor and determine biomechanical performances. Several system can be used for the motion capture, in the following we report two different 
solutions that were adopted at University of Cassino and Southern Lazio, for more information the reader can refer to (Rea et al., 2013a). The two proposed methods are used to monitor and acquire the movements of the body during the sit-to-stand, more specifically, by using a single camera, as reported in the illustrative example of Fig. 1 and 2, alternatively referring to an experimental apparatus bases on two cameras, as shown in Fig. 3 .

For both cases, the following procedure was used: Individuals were asked to be seated on an armless chair set to the $100 \%$ of the knee height. The back support of the chair was used to set the trunk in a vertical position. The arms did not participate actively to the movement.

The subjects were asked to assume a seated position of readiness and then they were asked to stand up from the chair. During the test the speed of the movement was kept constant, indeed in quasi-static conditions were obtained. Experimental tests were carried out at Cassino and Southern Lazio by considering healthy and young volunteers (age 20-30). Experimental results for the STS movements of female and male subjects are given in Fig. 1 and 2. The other proposed solution with two cameras makes use of the Virtual Sensei Lite, which is a free version of VS (2016). In this context, we used the software for the evaluation of the STS together with a motion capture system based on the cameras. Figure 3 shows the experimental set-up and result of a male subject, which is then used for the design of the support mechanisms.

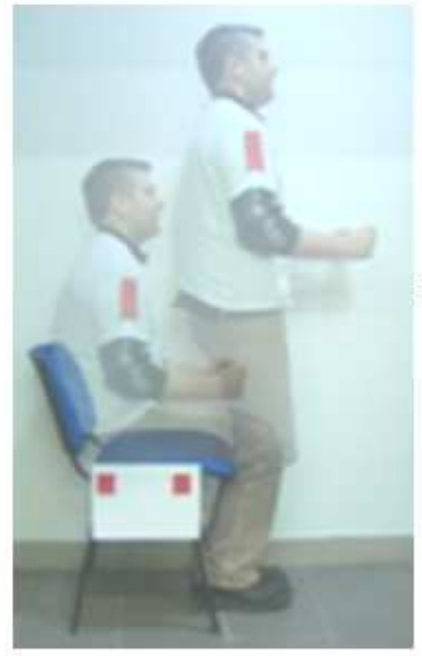

(a)

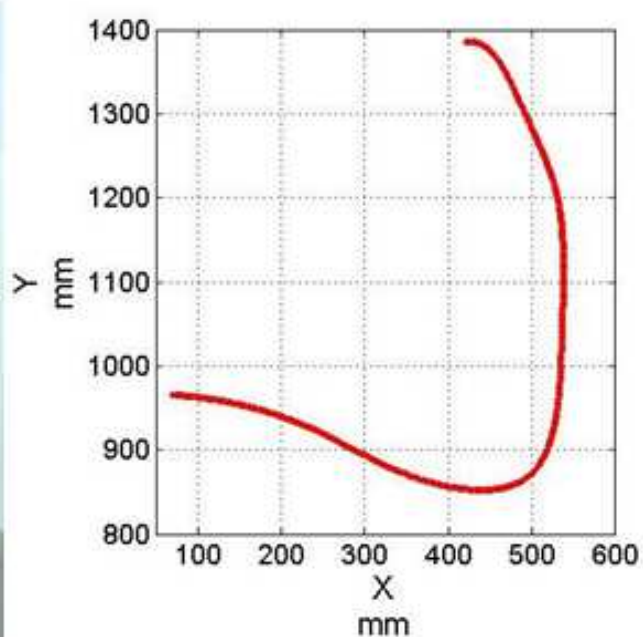

(b)

Fig. 1. Experimental set-up with a single camera: (a) experimental results for a male; (c) COG trajectory

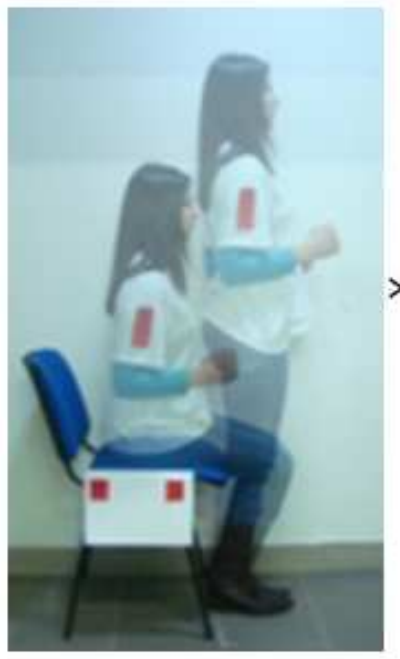

(a)

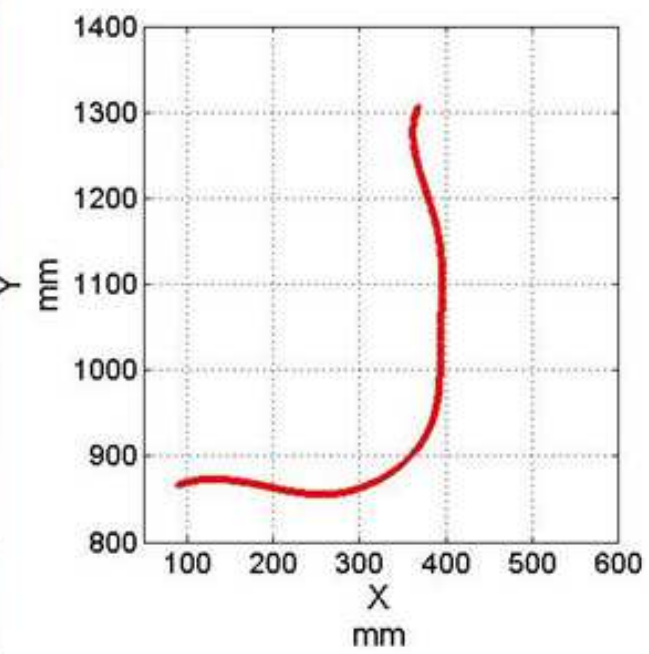

(b)

Fig. 2. Experimental set-up with a single camera: (a) experimental results for a female; (b) COG trajectory 


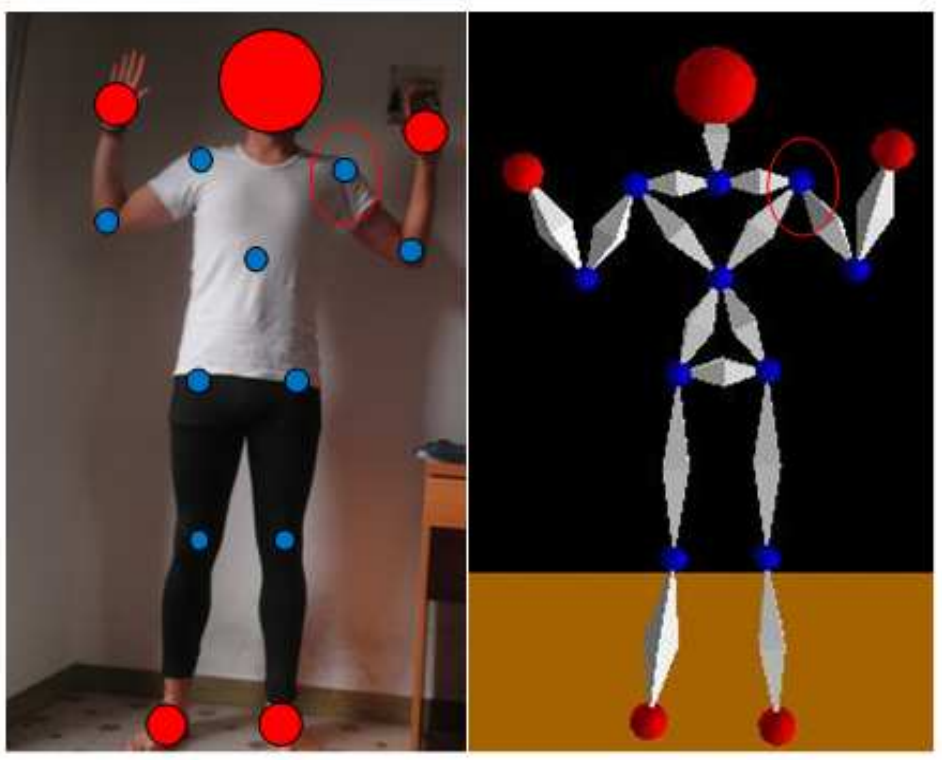

(a)

(b)

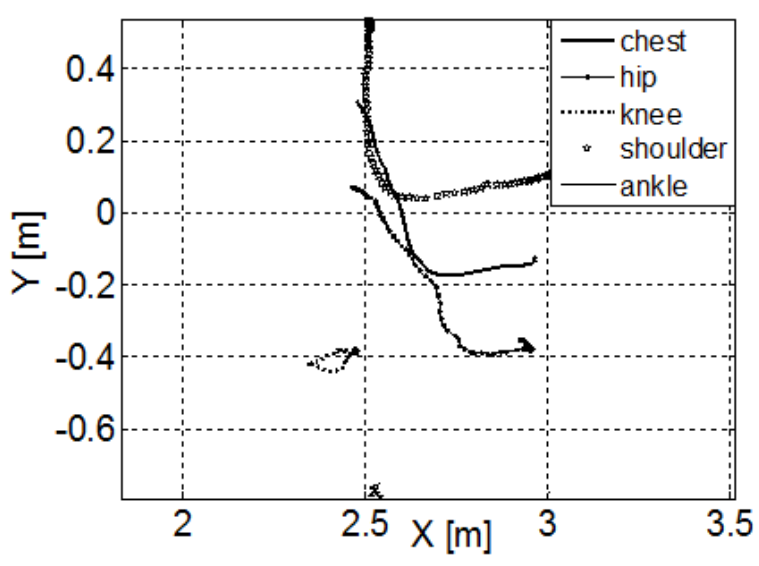

(c)

Fig. 3. Experimental set-up with two cameras: (a) Subject during the calibration; (b) image from Virtual Sensei Lite; (c) experimental results

\section{Mechanical Design Solutions for the STS}

For the study the sit-to-stand, the enormous variation of motion patters is one of the main issues, not only because of the differences in the anthropometric data of individuals, but also for any unique style of movement as distinctive of personality, age, weight strength in muscles. Furthermore, each individual will never repeat exactly the same movement from trial to trial or even in the same trial. Taking into account these issues, in our studies of the human movement and in developed procedures, we have considered either the COG trajectory (or other points of interest) and the orientation of the trunk. This will lead to considerations on the synthesis of the mechanism to support and sustain the body during the sit-to-stand.
Referring to the design of an assisting device, it is basically composed by a mechanical part, mainly the support mechanism, which will be responsible of the requested movement, an actuation system to give suitable power to lift the individual and interfaces to grab and sustain the body. In addition, any security system to prevent accidental falls and brake system should be included in the overall mechatronic design. A classification for the sit-to-stand mechanisms can be done as based on the number of Degrees of Freedom (DOFs) and actuation type. Focusing the attention on the mechanism devoted to reproduce a desired movement, the linkage has to fulfill the following requirements: The device should possess 2-DOFS in the sagittal plane to accomplish the requested trajectories, which vary according to anthropometric data of the subject. An 
additional DOF can be included to provide suitable orientation for the trunk support. According to these initial specifications, the designer should determine the topology of the kinematic chain underlying the mechanical structure, indeed choosing among serial, parallel or hybrid structures. If the requested motion lies in the sagittal plane, a planar mechanism instead of spatial one can be considered. The most complex and flexible solution is represented by 3 -DOF mechanisms to perform any trajectory in the sagittal plane having controllable orientation of the trunk support. Alternatively, the orientation of the trunk can be ignored or left adjustable manually, indeed not controllable, therefore, a 2-DOF mechanism to reproduce the COG trajectory can be chosen. The last option is to simplify the support mechanism by providing a given and not programmable trajectory of the COG and discarding the orientation of the trunk support. It is evident that the more the DOFs of the mechanism are, higher is the complexity of the control and the overall cost of the mechatronic device. If a simplified solution is chosen, the following advantages are encountered: (1) Easy-inuse system for non-expert generic users; (2) simpler control units and less expensive actuation system than 3DOFS systems. In addition, a large number of commercialized systems are lifters with just 1-DOF that lifts the person without giving any particular trajectory to the trunk. In the following, we describe three different solutions designed by taking into account experimental data obtained in the trial and reported in Fig. 3.

\section{Three DOF Mechanism}

The designed mechanism in Fig. 4 has been based on the topology reported in (Chugo et al., 2008).

The support mechnism is composed by a 2-DOF fivebar linkage ABCDEH, whose actuations are $M_{1}$ with $\theta_{1}$ and $M_{2}$ with $\theta_{2}$ and the linkage EFGH, whose actuation is $M_{3}$ with $\theta_{5}$ to provide suitable orientation to the trunk support. The device is completed by fixed supports for the arms. In order to solve the Direct Kinematic problem equations are described for the five-bar linkage $\mathrm{ABCDEH}$ in the form:

$$
\begin{gathered}
L_{1} \cos \theta_{1}+L_{3} \cos \theta_{3}+L_{4} \cos \theta_{4}+L_{2} \cos \theta_{2}=0 \\
L_{1} \sin \theta_{1}+L_{3} \sin \theta_{3}+L_{4} \sin \theta_{4}+L_{2} \sin \theta_{2}=0
\end{gathered}
$$

and for the four-bar linkage EFGH:

$$
\begin{aligned}
& L_{6} \cos \theta_{6}=-L_{5} \cos \theta_{5}-L_{7} \cos \theta_{7}-L_{4} \cos \theta_{4} \\
& L_{6} \sin \theta_{6}=-L_{5} \sin \theta_{5}-L_{7} \sin \theta_{7}-L_{4} \sin \theta_{4}
\end{aligned}
$$

For them the geometry of the system is given, together with the input joint angles $\theta_{1}, \theta_{2}$ and $\theta_{5}$. Equation 1 and 2 are used to obtain the remain angles.
Once that all angles are derived the position of point $\mathrm{P}$ can be described as function of the input joints and configuration of the support mechanism.

\section{Two DOF Mechanism}

The designed mechanism given in Fig. 5 is based on a double-pantograph, which gives the vertical motion, as it is commonly used as lifting system for industrial applications, together with a linear guide providing the horizontal motion. The advantages of this design solution are: (1) Planar mechanism, easy to design and manufacture, (2) totally decoupled motion, which reduces the complexity of control scheme of the system, (3) possibility to use linear motors. It has been chosen a double pantograph in order to use a smaller stroke of the linear motor for the vertical motion.

Figure $5 \mathrm{~b}$ shows a kinetostatic scheme for the designed mechanism, the reference frame has been fixed at point $O, q_{1}$ and $q_{2}$ are the input linear displacements for the actuators being $f(x, y)=0$ the implicit function that describes the desired trajectory. It is worth noting that, since the 2 DOFs are decoupled, the doublepantograph actuation $q_{1}$ and the second linear actuator $q_{2}$ can be considered separately.

In particular, in the following the position analysis of the first mechanism can be expressed as:

$y=2 h ; h=l \sin \vartheta ; q_{1}=l \cos \vartheta ; h^{2}=l^{2}-q_{1}^{2}$

Differentiating (3) with respect to time gives:

$\dot{y}=2 \dot{h} ; \dot{h}=l \cos \vartheta \dot{\vartheta} ; \dot{q}_{1}=-l \sin \vartheta \dot{\vartheta}$

Transmission ratio for the pantograph mechanism is:

$\tau=\frac{2}{\tan \vartheta}$

Assuming an almost constant mechanical efficiency $\eta$, neglecting links' masses and their moment of inertia, if a quasi-static movement is executed, then energy balance can be expressed as:

$Q \dot{q}_{1}=2 P \frac{\dot{h}}{\eta}$

In which $Q_{1}$ is the modulus of the actuation force at the double-pantograph mechanism, $P=m g$ is the modulus of payload, that is the body weight to be lifted. Therefore, the requested actuation force vary according to the transmission ratio of the mechanism as:

$Q_{1}=\tau \frac{m g}{\eta}$ 


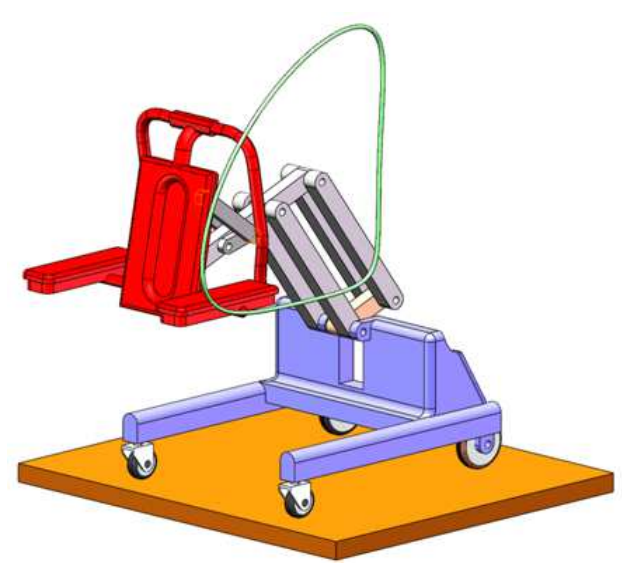

(a)

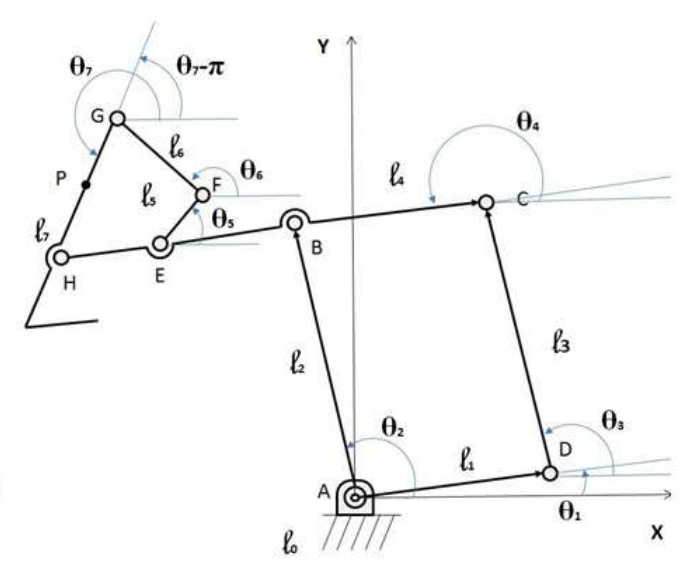

(b)

Fig. 4. 3-DOF mechanism for the generation of the trunk motion: (a) 3D sketch; (b) kinematic scheme

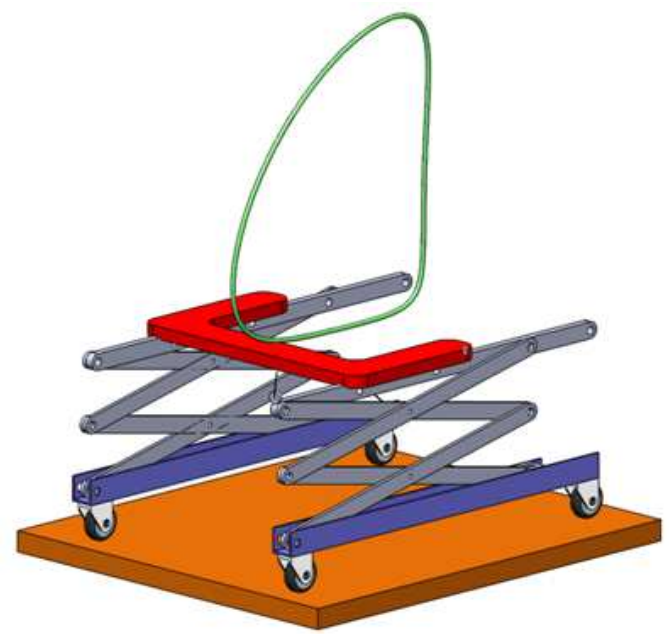

(a)

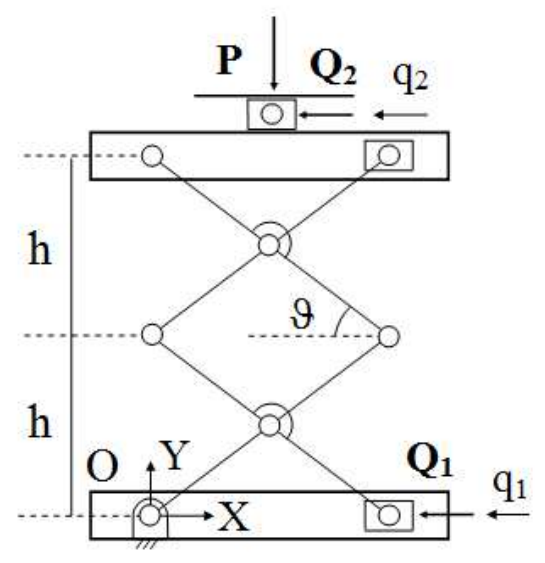

(b)

Fig. 5. Two DOF mechanism for the generation of the trunk motion: (a) 3D sketch; (b) kinematic scheme

In order to get design consideration we differentiate $\tau$ with respect to the vertical displacement

$$
\frac{\partial \tau}{\partial y}=-\frac{2 \sqrt{l^{2}+y^{2}}}{y}
$$

The value in Equation 8 is always negative, furthermore, $Q_{1}$ increases if $y$ decreases and it tends to infinity when $y$ tends to zero. Therefore, we should fix a minimum value for $y$ that is the mechanism cannot be completely folded when it is not used. It is worth to note that the system is represented in a skeleton form and the various mechanical parts could be further covered or encased. No such covering or encasement has been shown at this stage since it would merely obscure the operation of the working parts. Furthermore, it has been assumed that the interaction of the system with the end- user takes place through the armpit and then an interface similar to a crutch can be configured. Wrap around belts can be also provided to hold the torso and prevent antepulsion and retropulsion.

\section{One DOF Mechanism}

The support mechanism to accomplish the requested sit-to-stand motion can be also synthetized by using a 1DOF mechanism to reproduce the assigned rigid-body motion, as shown in Fig. 6.

In particular, a four-bar linkage has been chosen and the Burmester problem, which aims at finding its geometric parameters required for a prescribed set of finitely separated poses is solved for the case understudy. The equation of a coupler point curve is then obtained by analytic geometry being the loci of any point $\mathrm{P}$ that belongs to a segment for which points $\mathrm{A}$ and $\mathrm{B}$ are constrained to lie on two circles. 


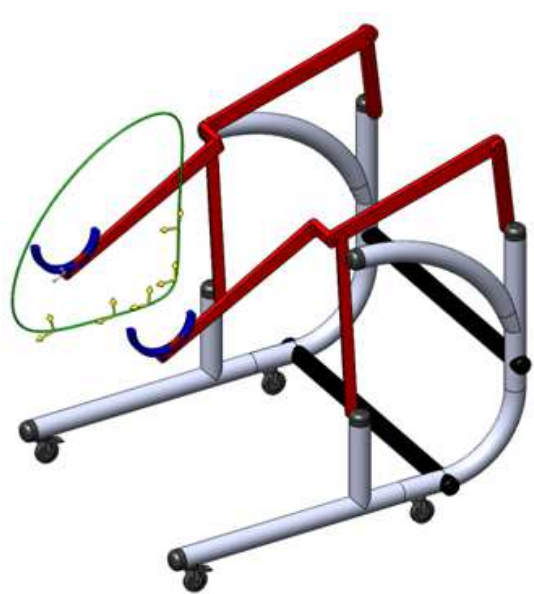

(a)

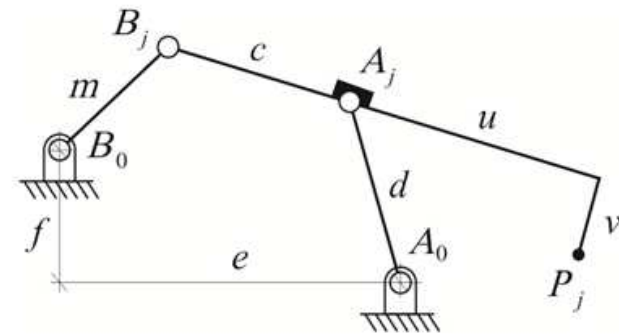

(b)

Fig. 6. One DOF mechanism for the generation of the trunk motion: (a) 3D sketch; (b) kinematic scheme

According to the kinematic scheme in Fig. 6b, the position of point $P$, with coordinates $(x, y)$ can be described with respect a fixed frame, as a function of kinematic parameters of the mechanism $A_{0} A B_{0} B P$.

Links $A_{0} A$ and $B_{0} B$ can be evaluated as:

$$
\left\|A_{0} A\right\|^{2}=d^{2} ;\left\|B_{0} B\right\|^{2}=m^{2}
$$

Which can be rewritten by considering the fixed frame attached to point $A_{0}$ as:

$$
\begin{aligned}
& A_{0} A=\left[\begin{array}{l}
x+u \cos \theta-v \sin \theta \\
y+u \sin \theta+v \cos \theta
\end{array}\right] \\
& B_{0} B=\left[\begin{array}{l}
x-e+(u+c) \cos \theta-v \sin \theta \\
y-f+(u+c) \sin \theta+v \cos \theta
\end{array}\right]
\end{aligned}
$$

Substituting Equation 10 in Equation 9, one can obtain two equations, which are function of design parameters and $x, y$ and $\theta$. The desired function can be obtained by eliminating $\theta$ and further algebraic manipulations. Details are reported in Hunt (1990) by using dialytic elimination method. Once that the problem is solved the resulting equation is the 6th order polynomial expression of point $P$ given in the fixed frame, when a single actuation is given at point $B_{0}$.

For this design solution the interaction between the system and the end-user takes place through the armpit, therefore, an interface similar to the previous case has to be considered.

\section{Simulation Results}

Simulation tests were carried out considering the 3 design solutions proposed in the previous section. In particular, Fig. 7 and 8 reports the simulation results of the 3-DOF mechanism design in Fig. 4 for the sit-to- stand by imposing the trajectory obtained during the experimental trial in Fig. 3. The trajectory is also reported in the scheme of Fig. 4a). It is worth noting that the orientation is fixed in order to compare the result with the ones obtained with the other solutions.

Therefore, plots in Fig. 7 and 8 refers to the actuation of the motors $M_{1}$ and $M_{2}$, discarding the third motor $M_{3}$, which is responsible to the change of the orientation of the trunk support.

Figure 9 refers to the design solution of Fig. 5 with the support mechanism having 2 active joints, which are two linear guides providing the horizontal and vertical motions. For representative purposes, the plots in Fig. 9a and $9 \mathrm{c}$ refers to the displacements of the two active joints during the sit-to-stand trajectory reported in the scheme of Fig. 5a and related to the experimental trial of Fig. 3.

Figure 10 shows numerical results of the simulation carried out with the 1-DOF mechanism shown in Fig. 6 and reproducing the trajectory in Fig. 3.

In particular, the angular velocity and the requested torque are displayed in Fig. 10 for the case understudy.

It is worth noting that the simulations were run under the same following circumstances, an individual is considered with a mass of $100 \mathrm{~kg}$, completely lying on the armpits support, which represents the worst case in which a person does not participate actively to the movement of the sit to stand and therefore he/she has to be completely lifted.

The experimental activity was carried out in order to get useful information for design and simulation purposes. In particular, the experimental test of the male subject in Fig. 3 was used for the synthesis of the all support mechanisms for the three design solutions proposed in this paper. The acquired trajectory and body orientation were also used for the simulated motions to get useful information for the actuation and control of the three design solutions. In particular, the following considerations arises. 
First, it is well known that increasing the number of DOFs of a systems or mechanism its flexibility increases to accomplish several tasks, like programming suitable trajectories according to the anthropometric data of the individual or modify a trajectory according to the changing of personal performances after a training period. This result can be achieved with 3 or 2 DOF mechanisms, as those proposed in this paper, having or not the possibility to change the orientation of the trunk.

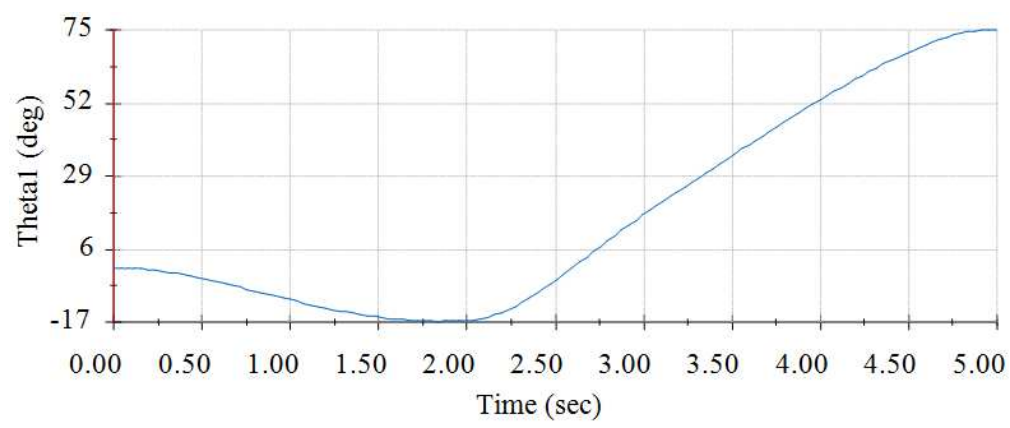

(a)

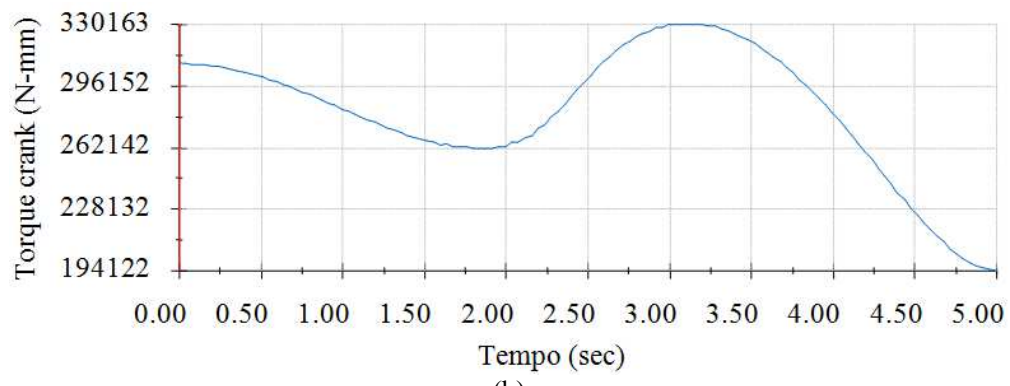

(b)

Fig. 7. Simulation results for the 3 DOF mechanism in Fig. 4 (motor M1): (a) rotation angle; (b) input torque

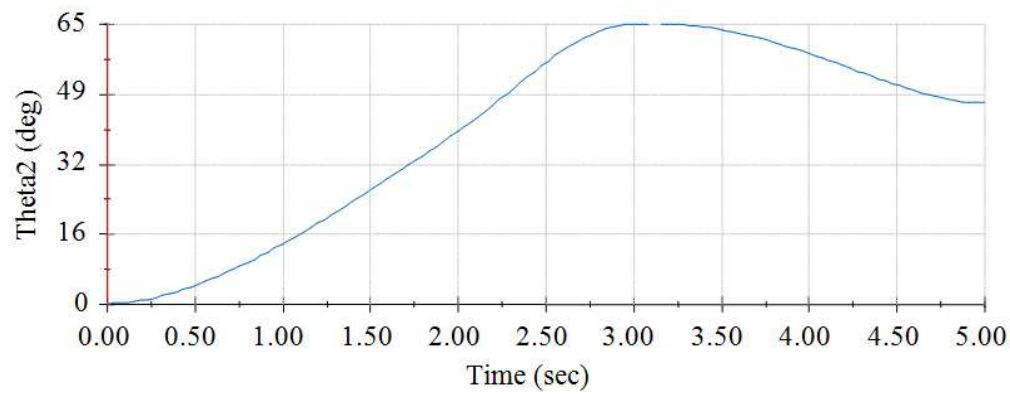

(a)

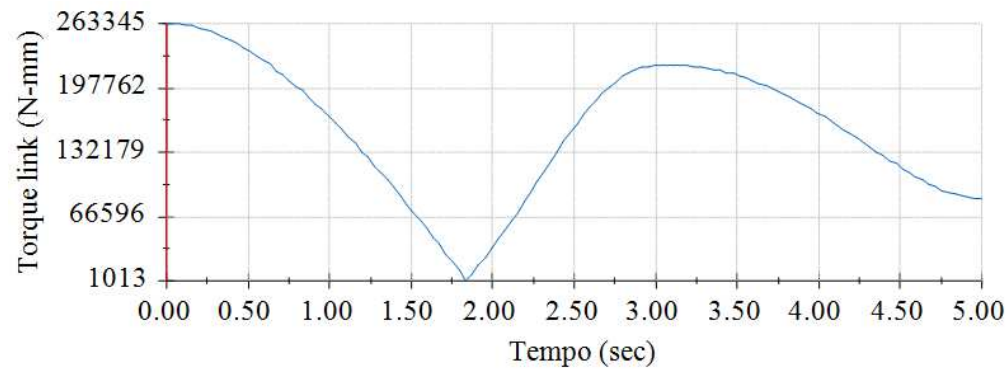

(b)

Fig. 8. Simulation results for the 3-DOF mechanism in Fig. 4 (motor M2): (a) rotation angle; (b) input torque 
Pierluigi Rea and Erika Ottaviano / American Journal of Engineering and Applied Sciences 2016, 9 (4): 1134.1143

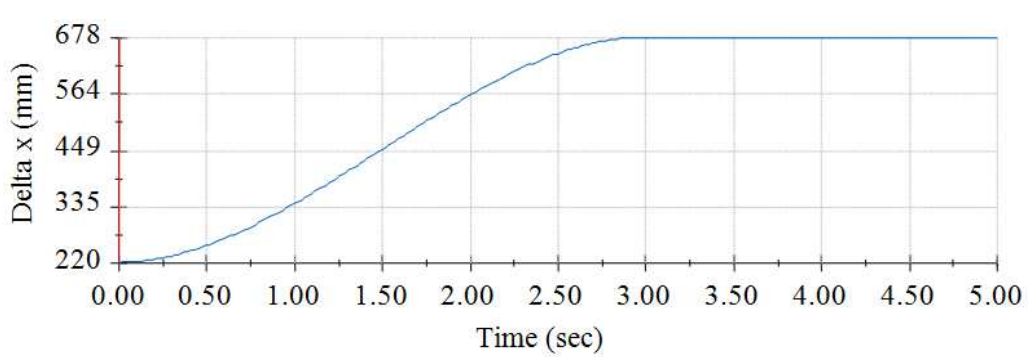

(a)

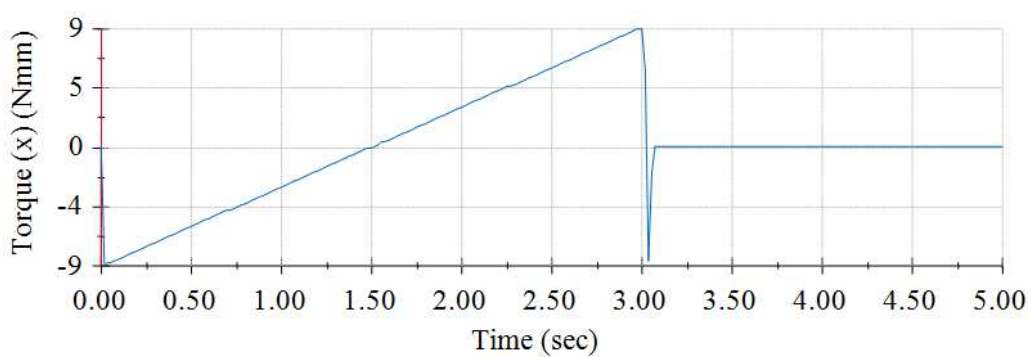

(b)

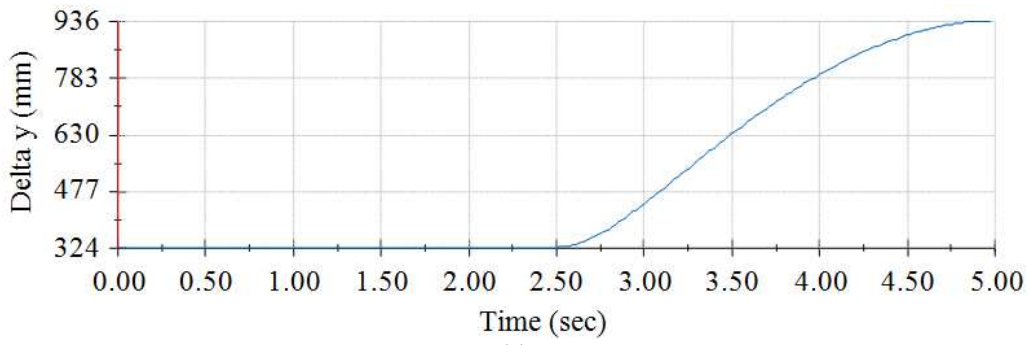

(c)

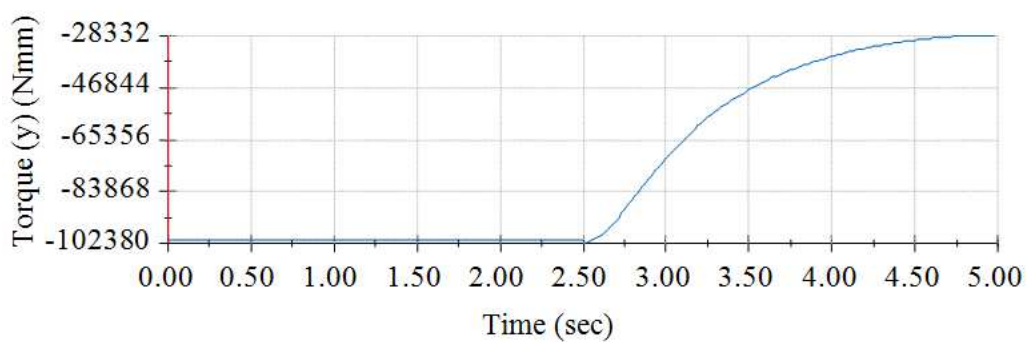

(d)

Fig. 9. Simulation results for the 2 DOF support mechanism in Fig. 5: (a) displacement along $x$; (b) torque of the motor to obtain the displacement along $\mathrm{x}$-axis; (b) displacement along y-axis; (d) torque of the motor to obtain the displacement along y-axis

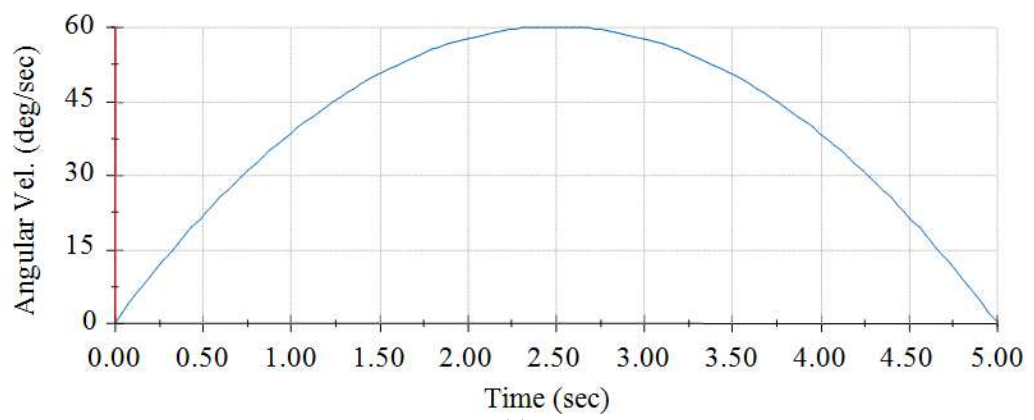

(a) 


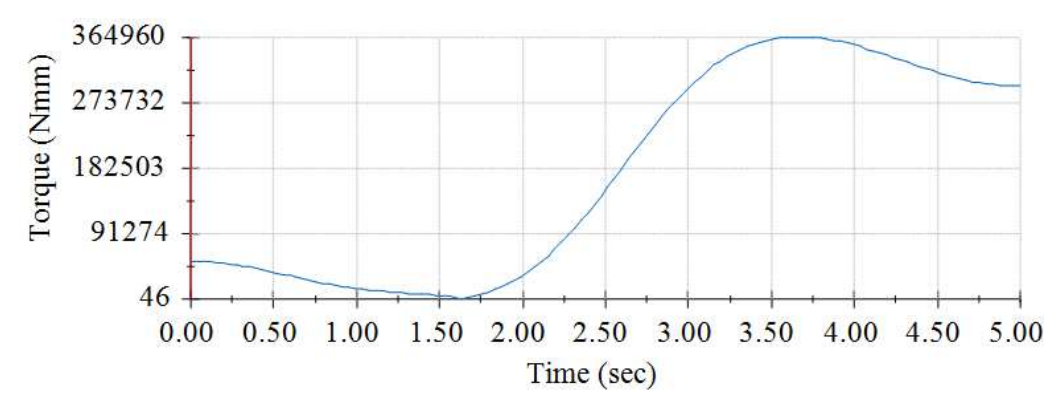

(b)

Fig. 10. Simulation results for the 1-DOF mechanism in Fig. 6: (a) angular velocity of the input joint; (b) input torque

For these reported cases, a control scheme and strategy can be developed. A HMI interface can be used to set parameters referring to an individual and his/her physical conditions. The information of a given trajectory acquired with a motion capture system as one of the two proposed in this paper can be processed by a motion control block implemented on a PC. A PLC connected with suitable Kinetix drives can send signals to drive the motor and can receive the feedback from the sensors. Thus, it is possible to obtain a suitable close-loop control in velocity or force (Figliolini and Rea, 2007). MP Series actuators by Rockwell Automation may be selected because they have favorable characteristics for the application.

The drawback of this kind of solution is mainly related to the increase of the system complexity in terms of costs of the overall system and control and coordination of the actuation.

The alternative of this kind of flexible solution customizable at the top by the end-used is to consider a simplified solution that has to be customized at the bottom, that is at the design stage. A single DOF mechanism can be synthetized at the design stage according to the given COG trajectory and then the resulting systems will be less expensive in terms of hardware and control, because a single motor has to be programmed. This design solution is the most compact and less expensive leading to possible applications for the homecare of elderly or individual with reduced mobility. The drawback of this solution is that the system can be customized at the very beginning leading to modifications only by changing the support mechanism. Summarizing, the examples of devices reported here show how the problem of the sit-to-stand can be faced using different types of solutions according to main specifications, starting with the same motion requirements. It should be pointed out that each solution reported here presents advantages but also drawbacks, therefore the choice has to be made taking into account all factors and intended use of the device.

\section{Conclusion}

Recently, due to the high demand for assistive technology and related solutions, there is a growth and rapid development of reliable, easy-to-use and rather economical systems for improving the quality of daily-life of individuals with reduced mobility. In this paper, we have analyzed three solutions for the design of assistive devices for the sit-to-stand, which is a change of posture from seated to standing. Comparative considerations are reported as based on the results of experimental tests and numerical simulations for the three cases of study in which 3, 2 or 1DOF mechanisms are designed and simulated.

\section{Acknowledgement}

Adult volunteers that took part to the experimental trial at University of Cassino and Southern Lazio are greatly acknowledged.

\section{Funding Information}

This is to declare that the paper was published without external funds.

\section{Author's Contributions}

All the authors contributed equally to prepare, develop and carry out this work.

\section{Ethics}

This article is original. Author declares that are not ethical issues that may arise after the publication of this manuscript.

\section{References}

Chugo, D., T. Asawa, T. Kitamura, S. Jia and K. Takase, 2008. A rehabilitation walker with standing and walking assistance. Proceedings of the IEEE/RSJ International Conference on Intelligent Robots and Systems, Sep. 22-26, IEEE Xplore Press, Japan, pp: 260-265. DOI: 10.1109/IROS.2008.4650845

Figliolini, G. and P. Rea, 2007. Ca.U.M.Ha. Robotic hand (Cassino-Underactuated-Multifinger-Hand). Proceedings of the IEEE/ASME International Conference on Advanced Intelligent Mechatronics, Sep. 4-7, IEEE Xplore Press, Italy, pp: 250-250. DOI: 10.1109/AIM.2007.4412562 
Graf, B., M. Hans and D.S. Rolf, 2004. Care-O-bot IIdevelopment of a next generation robotic home assistant. J. Aut. Ro., 16: 193-205.

DOI: 10.1023/B:AURO.0000016865.35796.e9

Hirata, Y., J. Higuchi, T. Hatsukari and K. Kosuge, 2008. Sit-to-stand assist system by using handrail and electric bed moving up and down. Proceedings of the 2nd IEEE RAS and EMBS International Conference on Biomedical Robotics and Biomechatronics, Oct. 19-22, IEEE Xplore Press, Sendai, pp: 187-192.

DOI: 10.1109/BIOROB.2008.4762786

Hunt, K.H., 1990. Kinematic Geometry of Mechanisms. 1st Edn., Oxford University Press, New York, ISBN-10: 0198561245.

Krishnan, R.H. and S. Pugazhenthi, 2014. Mobility assistive devices and self-transfer robotic systems for elderly, a review. Int. Ser. Rob., 7: 37-37. DOI: $10.1007 / \mathrm{s} 11370-013-0142-6$

Martins, M.M., C.P. Santos, A. Frizera-Neto and R. Ceres, 2012. Assistive mobility devices focusing on smart walkers: Classification and review. Rob. Auto. Syst., 60: 548-562.

DOI: 10.1016/j.robot.2011.11.015

Nagai, K., I. Nakanishi and H. Hanafusa, 2003. Assistance of self-transfer of patients using a powerassisting device. Proceedings of the IEEE International Conference on Robotics and Automation, Sep. 14-19, IEEE Xplore Press, Shiga, Japan, pp: 4008-4015.

DOI: 10.1109/ROBOT.2003.1242213
Palopoli, L., A. Argyros, J. Birchbauer, A. Colombo and D. Fontanelli et al., 2015. Navigation assistance and guidance of older adults across complex public spaces: The DALi approach. Int. Ser. Rob., 8: 77-92. DOI: $10.1007 / \mathrm{s} 11370-015-0169-\mathrm{y}$

Rea, P., E. Ottaviano and G. Castelli, 2013a. A Procedure for the design of novel assisting devices for the sit-to-stand. J. Bio. Eng., 10: 488-496. DOI: $10.1016 / \mathrm{S} 1672-6529(13) 60249-8$

Rea, P., E. Ottaviano, M. Conte, A. D'Aguanno and D. De Carolis, 2013b. The design of a novel tilt seat for inversion therapy. Int. J. Ima. Rob., 11: 1-10.

Schiffer, S., A. Ferrein and G. Lakemeyer, 2012. Caesar: An intelligent domestic service robot. Int. Service Robot., 5: 259-259. DOI: $10.1007 / \mathrm{s} 11370-012-0118-y$

SSB, 2016. SUNY Stony Brook.

EIU, 2011. The future of healthcare in Europe. The Economist Intelligence Unit.

TTW, 2016. Topro Taurus Walker.

VS, 2016. Virtual Sensei.

Yoshimitsu, T. and K. Yamamoto, 2004. Development of a power assist suit for nursing work. Proceedings of the Annual Conference, Aug. 4-6, IEEE Xplore Press, Japan, pp: 577-580. 